\title{
Hershberger Assays for Bisphenol-A and Its Substitute Candidates
}

\author{
Hee-Su Kim ${ }^{1}$, Yong-Bin Kim ${ }^{1}$, Donchan $\mathrm{Choi}^{2}$, Yong-Pil Cheon ${ }^{3}$ and ${ }^{\dagger}$ Sung-Ho Lee ${ }^{1}$ \\ ${ }^{1}$ Dept. of Biotechnology, Sangmyung University, Seoul 03016, Korea \\ ${ }^{2}$ Dept. of Life Science, Yong-In University, Yongin 17092, Korea \\ ${ }^{3}$ Division of Developmental Biology and Physiology, Sungshin University, Seoul 02844, Korea
}

\begin{abstract}
Bisphenol-A(BPA) is a member of alkylphenol family, and shows adverse effects including reduced fertility, reproductive tract abnormalities, metabolic disorder, cancer induction, neurotoxicity and immunotoxicity. In the present study, we conducted Hershberger assay to evaluate whether the two candidates to replace BPA have androgenic or antiandrogenic activity. The assay was carried out using immature castrated Sprague-Dawley male rats. After 7 days of the surgery, testosterone propionate (TP, $0.4 \mathrm{mg} / \mathrm{kg} /$ day) and test materials (low dose, $40 \mathrm{mg} / \mathrm{kg} / \mathrm{day}$; high dose, $400 \mathrm{mg} / \mathrm{kg} /$ day) were administered for 10 consecutive days by subcutaneous (s.c.) injection and oral gavage, respectively. Test materials were BPA, isosorbide (ISO) and cyclohexanedimethanol (CHDM). The rats were necropsied, and then the weights of five androgen-dependent tissues [ventral prostate, seminal vesicle, levator ani-bulbocavernosus (LABC) muscle, paired Cowper's glands, and glans penis] and three androgen-insensitive tissues (kidney, spleen and liver) were measured. All test materials including BPA did not exhibit any androgenic activity in the assay. On the contrary, antiandrogen-like activities were found in all test groups, and the order of the intensity was CHDM > BPA > ISO in the five androgen-sensitive tissues. There was no statistical difference between low dose treatment and high dose treatment of BPA group as well as ISO group. In CHDM group, high dose treatment exhibited most severe weight reduction in all measured tissues. There was no statistical difference in androgen-insensitive tissue measurements, except BPA groups. Since the effects of ISO treatment on the accessory sex organs were much less or not present at all when compared to those of BPA, ISO could be a strong candidate to replace BPA. CHDM treatment brought most severe weight reduction in all of androgen-sensitive tissues, so this material should be excluded for further screening of BPA substitute selection.
\end{abstract}

Key words : Rat, Hershberger assay, Bisphenol-A, Isosorbide (ISO), Substitute

\section{INTRODUCTION}

Endocrine disrupting chemicals or compounds (EDCs) are substances that can interfere with endocrine systems in animals including human. Among them, bisphenol-A(BPA) is a member of alkylphenol family and is used primarily to make plastics, and products using BPA-based plastics have been available in commercial since 1957 (Nimrod \& Ben- son, 1996; Pivnenko et al., 2015). More recently, BPA is a preferred color developer in carbonless copy paper and thermal point of sale receipt paper (Karwacka et al., 2017). Actually, BPA seems to be most commonly exposed industrial EDC in modern society, and its adverse effects have been extensively studied over past two decades (Giulivo et al., 2016; McLachlan, 2016).

Numerous studies have demonstrated an association

\footnotetext{
Manuscript received October 21, 2017, Received in revised form November 26, 2017, Accepted December 10, 2017

${ }^{\dagger}$ Corresponding Author : Sung-Ho Lee, Ph.D., Department of Biotechnology, Sangmyung University, Hongjimoon-2 gil 20, Jongrho-Gu, Seoul 03016, Republic of Korea. Tel: +82-2-2287-5139, Fax: +82-2-2287-0070, E-mail: shlee@ smu.ac.kr

This is an Open Access article distributed under the terms of the Creative Commons Attribution Non-Commercial License (http:// creativecommons.org/licenses/by-nc/3.0) which permits unrestricted non-commercial use, distribution, and reproduction in any medium, provided the original work is properly cited.
} 
between BPA expose and reproductive toxicities including abnormal reproductive tract formation, lower fertility, sign of early puberty and longer estrus, and abnormal breast development (Richter et al., 2007; Costa et al., 2014; Giulivo et al., 2016; Sifakis et al., 2017). BPA can mimic the structure and function of the hormone estradiol by binding to and activating the same estrogen receptors (ERs) as the natural hormone (Dickerson \& Gore, 2007). Although most of BPA effects have been found in female, a considerable amount of research revealed a connection between BPA expose and male reproductive disorders such as altered prostate cell physiology and sperm abnormalities (Ho et al., 2006; Manfo et al., 2014).

Due to many detrimental effects of BPA, particularly on the fetus and newborn (Meeker, 2012), the task making plastic products with BPA alternatives becomes much more urgent. Consequently, the potential negative effects of the candidates should be accurately and efficiently screened. So far, there are two standard evaluation procedures recommended by Environmental Protection Agency (EPA) and Organisation for Economic Co-operation and Development (OECD) for initial in vivo screening of compounds with estrogenic or androgenic nature; Uterotrophic assay and Hershberger assay, respectively.

The Hershberger Bioassay is a short-term in vivo screening test using accessory tissues of the male reproductive tract to detect compounds with potential to exert androgenic or antiandrogenic activities (EDSP, 1998; OECD, $2007,2008)$. This assay is relatively convenient and reliable because five androgen-dependent organs, in castrated rats, sensitively respond to chemical with androgenic or antiandrogenic activities as an increase or decrease in absolute weights. The Hershberger assay have been thought to be sensitive and specific for androgenic responses and, though it is little bit complicated, also sensitive and specific for androgenic responses (Marty \& O’Connor, 2014).

In the present study, we conducted Hershberger assay to evaluate whether the two candidates to replace BPA have androgenic or antiandrogenic activity.

\section{MATERIALS AND METHODS}

\section{Chemicals}

Testosterone propionate (TP), bisphenol-A (BPA, CAS number 80-05-7), Isosorbide (ISO, CAS number 652-67-5) and cyclohexanedimethanol (CHDM, CAS number 10508-8) and corn oil were purchased from Sigma Chemical Co. (St. Louis, MO, USA). All substances were stored in glass containers at room temperature.

\section{Animals and ethics approval}

Male Sprague-Dawley rats (28 days old) were obtained from DBL (Chungchungbuk-do, Korea) and reared in Sangmyung university animal facility under specific pathogen free (SPF)-conditions of 12-h light/dark cycle (lights on at 07:00 h) and constant temperature of $22 \pm 1{ }^{\circ} \mathrm{C}$. Rats had free access to normal chow (Purina, Seoul, Korea) and tap water ad libitum. All procedures used were approved by the Animal Care and Use Committee (2017-01-03) at Sangmyung University. All the animals were handled in accordance with the guidelines for animal experiments of the Association for Assessment and Accreditation of Laboratory Animal Care (AAALAC).

\section{Surgery and test substance administrations}

Rats were castrated (ORX) on postnatal day (PND) 42 under anesthesia by placing an incision in the scrotum and removing both testes and epididymides with a ligation of the blood vessels and seminal ducts. The animals were allowed to recover from castration for 7 days, and then animals were randomly assigned to different groups in terms of their body weights on PND $49(\mathrm{n}=6)$. TP was daily administered for 10 consecutive days by subcutaneous (s.c.) injection at a dose of 0.4 $\mathrm{mg} / \mathrm{kg}$ bw/day (dissolved in $0.5 \mathrm{~mL}$ corn oil). The route of 
administration for the test substances (low dose, $40 \mathrm{mg} / 5$ $\mathrm{mL} / \mathrm{kg}$ bw; high dose, $400 \mathrm{mg} / 5 \mathrm{~mL} / \mathrm{kg} \mathrm{bw}$ ) was via oral gavage.

For the androgenic activity test, the vehicle group (ORX) was the negative control, and the TP-treated group (ORX+ TP) was the positive control. The weights of the tissues were compared to the controls for statistically significant changes. Two dose groups of the test substance plus positive and vehicle (negative) controls were normally sufficient to determine if a chemical is an androgen agonist, and this design was therefore preferred for animal welfare reasons (EDSP, 1998).

\section{Sample collections}

Approximately 24 hours after the final administration of the test substance, the rats were necropsied according to standard laboratory procedures. As critical indices, the weights of five androgen-dependent tissues [ventral prostate, seminal vesicle, coagulating glands, levator anibulbocavernosus (LABC) muscle, paired Cowper's glands, and glans penis] were measured. The weights of optional tissues (kidney, spleen and liver) were also measured.

\section{Statistical analysis}

All values were expressed as mean S.E. $(n=6)$. Absolute tissues weights were analyzed using analysis of covariance (ANOVA) or Student's $t$-test. Data were expressed as means \pm S.E., and $P$ value $<0.05$ denoted the statistically significant difference.

\section{RESULTS}

No abnormal clinical wounds or body weight changes were observed in the rats administered with the test chemicals.

\section{Effects on androgen-dependent tissues}

The effects of BPA and replacement candidates on changes in body weights and androgen-dependent tissues weights in castrated male rats are shown in Table 1. In all organs, the most severe weight loss was commonly found in CHDM-400+TP group.

\section{1) Body weights}

Significant decreases in body weight were observed in all the BPA treatment groups except high dose group (BPA-400) when compared to their negative (ORX) and positive group $(\mathrm{ORX}+\mathrm{TP})$, respectively. However, comparison between control groups and ISO groups showed no significant changes. In CHDM groups, significant change was found only in low dose group (CHDM-40) compared to control group.

\section{2) Weights of $L A B C$}

Like body weight changes, significant decreases in LABC weight were observed in all the BPA treatment groups except high dose group (BPA-400). Co-treatment with ISO and TP groups induced significant changes, but the decrements were minimal compared to the BPA cotreated groups. In contrast, co-treatment with CHDM and TP groups exerted similar or stronger negative effects compared to BPA groups.

\section{3) Weights of glan penis}

Significant decreases in glan penis weight were observed in low dose BPA treatment groups (BPA-40 and BPA-40+ $\mathrm{TP})$. There was no significant change in ISO groups while all CHDM group except CHDM-40+TP group showed significant decreases.

\section{4) Weights of paired Cowper's glands}

Significant decreases in Cowper's gland weight were observed in BPA-40 group and BPA-400+TP group. There was no significant change in ISO groups while all CHDM group except CHDM-40 group shown significant decreases. 
Table 1. Effects of orally administered bisphenol A (BPA), Isosorbide (ISO) and cyclohexanedimethanol (CHDM) and subcutaneously administered testosterone propionate (TP) on body and androgen-sensitive organ weights of castrated rats

\begin{tabular}{lcccccc}
\hline \hline \multicolumn{1}{c}{ Treatment ${ }^{\mathrm{a}}$} & $\begin{array}{c}\text { Body } \\
\text { weight(g) }\end{array}$ & LABC(mg) & Penis(mg) & $\begin{array}{c}\text { Cowper's } \\
\text { glands(mg) }\end{array}$ & S.V.(mg) & Prostate(mg) \\
\hline INT & $267.9 \pm 12.5$ & $646.1 \pm 22.8$ & $262.2 \pm 12.3$ & $36.0 \pm 1.8$ & $226.3 \pm 11.8$ & $130.7 \pm 5.6$ \\
ORX & $275.8 \pm 7.4$ & $230.2 \pm 21.4$ & $151.0 \pm 10.6$ & $4.6 \pm 0.5$ & $28.5 \pm 2.4$ & $12.6 \pm 1.2$ \\
ORX+TP & $307.3 \pm 7.3$ & $553.2 \pm 17.5$ & $227.5 \pm 7.7$ & $19.0 \pm 0.8$ & $141.2 \pm 7.7$ & $75.0 \pm 4.9$ \\
BPA-40 & $240.0 \pm 5.1^{* *}$ & $161.0 \pm 7.7^{*}$ & $117.3 \pm 4.4^{*}$ & $3.3 \pm 0.2^{*}$ & $22.8 \pm 1.3^{*}$ & $8.1 \pm 0.5^{* *}$ \\
BPA-40+TP & $255.3 \pm 8.3^{\dagger \dagger}$ & $459.4 \pm 13.4^{\dagger \dagger}$ & $207.4 \pm 4.1 \dagger$ & $19.1 \pm 1.1$ & $140.9 \pm 4.1$ & $69.0 \pm 4.0$ \\
BPA-400 & $254.7 \pm 6.6$ & $209.1 \pm 9.7$ & $132.5 \pm 5.1$ & $3.7 \pm 0.4$ & $27.5 \pm 1.2$ & $11.3 \pm 0.5$ \\
BPA-400+TP & $273.1 \pm 5.9^{\dagger \dagger}$ & $463.3 \pm 13.5^{\dagger \dagger}$ & $514.8 \pm 7.7$ & $15.1 \pm 0.8^{\dagger \dagger}$ & $131.3 \pm 5.2$ & $71.6 \pm 2.7$ \\
ISO-40 & $266.3 \pm 4.0$ & $251.6 \pm 14.0$ & $151.1 \pm 3.6$ & $5.9 \pm 0.7$ & $31.1 \pm 2.8$ & $10.2 \pm 0.8$ \\
ISO-40+TP & $284.8 \pm 7.9$ & $485.5 \pm 21.6^{\dagger}$ & $212.1 \pm 3.1$ & $17.5 \pm 1.1$ & $127.7 \pm 12.2$ & $51.0 \pm 1.9^{\dagger \dagger \dagger}$ \\
ISO-400 & $281.5 \pm 9.0$ & $238.6 \pm 11.3$ & $151.0 \pm 6.1$ & $5.6 \pm 0.3$ & $25.3 \pm 2.1$ & $11.2 \pm 0.8$ \\
ISO-400+TP & $285.6 \pm 7.2$ & $491.7 \pm 3.8^{\dagger \dagger}$ & $224.2 \pm 6.6$ & $19.2 \pm 0.5$ & $142.0 \pm 5.4$ & $70.9 \pm 1.7$ \\
CHDM-40 & $254.8 \pm 5.1^{*}$ & $165.9 \pm 19.2^{*}$ & $124.4 \pm 4.7^{*}$ & $3.7 \pm 0.3$ & $19.7 \pm 1.2^{* *}$ & $8.1 \pm 0.3^{* *}$ \\
CHDM-40+TP & $293.7 \pm 6.4$ & $450.7 \pm 16.2^{\dagger \dagger}$ & $219.5 \pm 6.0$ & $13.1 \pm 1.0^{\dagger \dagger \dagger}$ & $112.8 \pm 3.5^{\dagger \dagger}$ & $56.5 \pm 3.6^{\dagger \dagger}$ \\
CHDM-400 & $274.6 \pm 8.3$ & $170.3 \pm 14.3^{*}$ & $122.1 \pm 4.2^{*}$ & $3.5 \pm 0.2^{*}$ & $17.4 \pm 1.0^{* * *}$ & $9.2 \pm 0.7^{*}$ \\
CHDM-400+TP & $290.9 \pm 6.6$ & $398.6 \pm 21.5^{\dagger \dagger \dagger}$ & $187.8 \pm 8.9^{\dagger \dagger}$ & $10.8 \pm 0.9^{\dagger \dagger \dagger}$ & $82.5 \pm 5.3^{\dagger \dagger \dagger}$ & $36.5 \pm 3.7^{\dagger \dagger \dagger}$ \\
\hline
\end{tabular}

${ }^{a}$ Castrated immature rats were administered with TP by subcutaneous injection for 10 days. One day after the final treatment, the accessory sex organs were removed carefully and weighed separately.

Symbols ${ }^{*},{ }^{* *}$ and ${ }^{* * *}$ meant significant difference in comparison between ORX group and test material group. ${ }^{*}, P<0.05 ;{ }^{* *}$, $P<0.01 ;{ }^{* * *}, P<0.001$.

Symbols ${ }^{+,++}$and ${ }^{+++}$meant significant difference in comparison between ORX group and test material group. ${ }^{\dagger}, P<0.05 ;{ }^{\dagger}$, $P<0.01 ;$ ††,$P<0.001$.

\section{5) Weights of seminal vesicle}

No significant change was observed in BPA groups except BPA-40 group. There was no significant change in ISO groups while all CHDM group showed significant decreases.

\section{6) Weights of ventral prostate}

No significant change in ventral prostate weight was observed in all the BPA and ISO groups except BPA-40 and ISO-40+TP groups. However, the weights of all the
CHDM groups were significantly decreased.

\section{Effects on androgen-independent organs}

The effects of BPA and replacement candidates on changes in optional organs (liver, kidney and spleen) weights in castrated male rats are shown in Tables 2.

\section{1) Weights of kidney}

Significant decreases in kidney weight were observed in all BPA treatment groups when compared to their negative 
Table 2. Effects of orally administered bisphenol A (BPA), isosorbide (ISO) and cyclohexanedimethanol (CHDM) and subcutaneously administered testosterone propionate (TP) on androgen-insensitive organ weights of castrated rats

\begin{tabular}{lccc}
\hline \hline Treatment & Kidney $(\mathrm{mg})$ & Spleen $(\mathrm{mg})$ & Liver $(\mathrm{g})$ \\
\hline INT & $1,117.8 \pm 17.1$ & $696.1 \pm 33.7$ & $11.0 \pm 0.4$ \\
ORX & $1,063.9 \pm 16.1$ & $688.1 \pm 34.1$ & $11.3 \pm 0.5$ \\
ORX+TP & $1,098.3 \pm 18.1$ & $895.4 \pm 55.8$ & $12.2 \pm 0.4$ \\
BPA-40 & $879.7 \pm 9.5^{* * *}$ & $634.0 \pm 25.6$ & $9.2 \pm 0.3^{* *}$ \\
BPA-40+TP & $1,006.4 \pm 15.6^{\dagger \dagger \dagger}$ & $746.6 \pm 59.1$ & $11.0 \pm 5.2$ \\
BPA-400 & $935.6 \pm 25.3^{* * *}$ & $625.3 \pm 43.2$ & $10.1 \pm 0.4$ \\
BPA-400+TP & $1,044.2 \pm 17.7^{\dagger}$ & $629.0 \pm 20.7^{\dagger \dagger}$ & $10.9 \pm 0.3^{\dagger}$ \\
ISO-40 & $1,045.5 \pm 18.9$ & $803.9 \pm 71.6$ & $10.2 \pm 0.2$ \\
ISO-40+TP & $1,092.7 \pm 22.7$ & $720.5 \pm 21.1 \dagger$ & $10.8 \pm 0.4^{\dagger}$ \\
ISO-400 & $1,038.1 \pm 26.7$ & $744.6 \pm 31.5$ & $11.2 \pm 0.5$ \\
ISO-400+TP & $1,111.4 \pm 29.5$ & $794.2 \pm 48.3$ & $11.8 \pm 0.4$ \\
CHDM-40 & $965.6 \pm 21.2^{* *}$ & $666.8 \pm 28.0$ & $10.1 \pm 0.3$ \\
CHDM-40+TP & $1,117.7 \pm 14.5$ & $761.6 \pm 38.2$ & $12.3 \pm 0.3$ \\
CHDM-400 & $1,036.0 \pm 38.7$ & $706.2 \pm 33.7$ & $11.0 \pm 0.4$ \\
CHDM-400+TP & $1,164.0 \pm 20.9^{\dagger}$ & $789.6 \pm 47.9$ & $12.4 \pm 0.4$ \\
\hline
\end{tabular}

${ }^{a}$ Castrated immature rats were administered with TP by subcutaneous injection for 10 days. One day after the final treatment, the accessory sex organs were removed carefully and weighed separately.

Symbols ${ }^{*},{ }^{* *}$ and ${ }^{* * *}$ meant significant difference in comparison between ORX group and test material group. ${ }^{*}, P<0.05$; ${ }^{* *}, P<0.01 ;{ }^{* * *}, P<0.001$.

Symbols ${ }^{+,++}$and ${ }^{+++}$meant significant difference in comparison between ORX group and test material group. ${ }^{\dagger}, P<0.05$; ${ }^{\dagger}, P<0.01 ;{ }^{\dagger \dagger}, P<0.001$.

control and positive control, respectively. However, comparison between control groups and ISO groups exerted no significant changes. In CHDM groups, significant change was found in CHDM-40 group and CHDM-400+TP group compared to control group.

\section{2) Weights of spleen}

Unlike kidney weight change, no significant change in spleen weight was observed in all the BPA group except
BPA-400+TP group. Also, there was no significant weight change in all ISO group and CHDM group except ISO40+TP group.

\section{3) Weights of liver}

Significant decreases in liver weight were observed in BPA-40 group and BPA-400+TP group. As spleen weights, there was no significant weight change in all ISO group and CHDM group except ISO-40+TP group. 


\section{DISCUSSION}

The extended definition of EDC is a structurally diverse class of synthetic and natural compounds that possess the ability to alter various component of the endocrine system and potentially induce adverse effects in exposed individuals and populations (Devillers et al., 2006). Indeed, EDCs encompass wide range of synthetic and natural compounds, and the list is getting longer. Simultaneously, a body of evidence on the adverse effects of EDCs is getting larger, so EDCs-related health issues have become a matter of global concern (WHO, 2013). For example, BPA is one of the highest volume chemicals produced worldwide since 1957, and this compound is frequently used for food and beverage storage containers and thermal paper (Rubin, 2011). Like other alkylphenol family members (e.g. octylphenol and nonylphenol), BPA shows xenoestrogen exhibiting estrogen-mimicking properties (Nimrod \& Benson, 1996). Numerous studies have demonstrated adverse health effects attributed to BPA including a range of reproductive problems such are reduced fertility, male and female reproductive tract abnormalities, metabolic disorder, cancer induction, neurotoxicity and immunotoxicity (Richter et al., 2007; Manfo et al., 2014; Karwacka et al., 2017; Giulivo et al., 2016; Ho et al., 2006; Itoh et al., 2012; Kimber, 2017).

Due to these BPA associated negative health effects, structural analogues, such as bisphenol S (BPS) have been introduced into the market and many substitutes are planned to be launched. However, little is known about the presence and toxicity of these emerging replacements, and concern has risen about them (Björnsdotter et al., 2017). Indeed, recent studies clearly demonstrated that two such BPA substitutes, BPS and bisphenol F (BPF), are hormonally active as BPA and have similar endocrine-disrupting effects (Eladak et al., 2015; Rochester \& Bolden, 2015). In this context, development of standard evaluation procedure(s) being able to screen the substitute candidates with good accuracy and efficiency is high priority need.

Recent studies on the BPA actions revealed that interpretation of the Hershberger or uterotrophic assay results could be tangled job. For example, emerging data show that BPA binds with a significant number of hormone receptors, including a number of nuclear and membranebound estrogen receptors, androgen receptors, as well as the thyroid hormone receptor, glucocorticoid receptor, and peroxisome proliferator-activated receptor gamma (PPAR $\gamma$ ) (Teng et al., 2013; Yoon et al., 2014; MacKay \& Abizaid, 2017). In particular, MacKay \& Abizaid (2017) postulated that this increased diversity of receptor targets may allow to explain the puzzling aspects of BPA and other EDCs pharmacology. The weight reduction effects by antiandrogen-like activities of BPA and test materials in the present study could not be originated from a single receptor activation but from multiple ones.

In the present study, we found that all test materials including BPA did not exhibit any androgenic activity in the Hershberger assay. The lack of androgenicity and weight-loss effects of BPA in the present study are in good agreement with previous reports (Kim et al., 2002; Nishino et al., 2006). Rather, antiandrogen-like activities were found in all test materials, and the order of the intensity was CHDM $>$ BPA $>$ ISO in 5 androgen-sensitive organs. There is no available information on the action mechanism of ISO and CHDM (i.e. their receptors), so we tentatively concluded the weight reduction effects of these materials are antiandrogen-like activities. Meanwhile, no androgenic or antiandrogenic activities of the test materials were found in the androgen-insensitive organs used in this study. In general, the effects of ISO treatment on the accessory sex organs were much less or not present at all when compared to those of BPA. Therefore, at this level of screening, ISO could be a BPA substitute. CHDM treatment brought most severe weight reduction in all of androgensensitive organs, so this material should be excluded for 
further screening of BPA substitute selection.

\section{ACKNOWLEDGEMENTS}

This study was supported by a grant of National Research Foundation of Korea (NRF-2015M3A9D7067365).

\section{REFERENCES}

Björnsdotter MK, de Boer J, Ballesteros-Gómez A (2017) Bisphenol A and replacements in thermal paper: A review. Chemosphere 182:691-706.

Costa EM, Spritzer PM, Hohl A, Bachega TA (2014) Effects of endocrine disruptors in the development of the female reproductive tract. Arq Bras Endocrinol Metabol 58:153-161.

Devillers J, Marchand-Geneste N, Carpy A, Porcher JM (2006) SAR and QSAR modeling of endocrine disruptors. SAR QSAR Environ Res 17:393-412.

Dickerson SM, Gore AC (2007) Estrogenic environmental endocrine-disrupting chemical effects on reproductive neuroendocrine function and dysfunction across the life cycle. Rev Endocr Metab Disord 8:143-159.

Eladak S, Grisin T, Moison D, Guerquin MJ, N'TumbaByn T, Pozzi-Gaudin S, Benachi A, Livera G, RouillerFabre V, Habert R (2015). A new chapter in the bisphenol A story: bisphenol $\mathrm{S}$ and bisphenol $\mathrm{F}$ are not safe alternatives to this compound. Fertil Steril 103:1121.

Giulivo M, Lopez de Alda M, Capri E, Barceló D (2016) Human exposure to endocrine disrupting compounds: Their role in reproductive systems, metabolic syndrome and breast cancer. A review. Environ Res 151:251-264.

EDSP (1998) Endocrine Disruptor Screening Program (EDSP) Hershberger Assay OCSPP Guideline 890.1400 Standard Evaluation Procedure (SEP). US Environmental Protection Agency.

Ho SM, Tang WY, Belmonte de Frausto J, Prins GS (2006).
Developmental exposure to estradiol and bisphenol A increases susceptibility to prostate carcinogenesis and epigenetically regulates phosphodiesterase type 4 variant 4. Cancer Res 66:5624-5632.

Itoh K, Yaoi T, Fushiki S (2012) Bisphenol A, an endocrine-disrupting chemical, and brain development. Neuropathology. 32:447-457.

Karwacka A, Zamkowska D, Radwan M, Jurewicz J (2017) Exposure to modern, widespread environmental endocrine disrupting chemicals and their effect on the reproductive potential of women: an overview of current epidemiological evidence. Hum Fertil (Camb) 31:1-24.

Kim HS, Han SY, Kim TS, Kwack SJ, Lee RD, Kim IY, Seok JH, Lee BM, Yoo SD, Park KL (2002) No androgenic/anti-androgenic effects of bisphenol-A in Hershberger assay using immature castrated rats. Toxicol Lett 135:111-123.

Kimber I (2017) Bisphenol A and immunotoxic potential: A commentary. Regul Toxicol Pharmacol 90:358-363.

McLachlan JA (2016) Environmental signaling: from environmental estrogens to endocrine-disrupting chemicals and beyond. Andrology 4:684-694.

MacKay H, Abizaid A (2017) A plurality of molecular targets: The receptor ecosystem for bisphenol-A (BPA). Horm Behav. 2017 Nov 14. pii: S0018-506X(17)303112. doi: 10.1016/j.yhbeh.2017.11.001. [Epub ahead of print]

Manfo FP, Jubendradass R, Nantia EA, Moundipa PF, Mathur PP (2014) Adverse effects of bisphenol A on male reproductive function. Rev Environ Contam Toxicol 228:57-82.

Marty MS, O'Connor JC (2014) Key learnings from the Endocrine Disruptor Screening Program (EDSP) Tier 1 rodent uterotrophic and Hershberger assays. Birth Defects Res B Dev Reprod Toxicol 101:63-79.

Meeker JD (2012) Exposure to environmental endocrine disruptors and child development. Arch Pediatr Adolesc Med 166:952-958.

Nimrod AC, Benson WH (1996) Environmental estrogenic 
effects of alkylphenol ethoxylates. Crit Rev Toxicol 26: 335-364.

Nishino T, Wedel T, Schmitt O, Schönfelder M, Hirtreiter C, Schulz T, Kühnel W, Michna H (2006) The xenoestrogen bisphenol A in the Hershberger assay: androgen receptor regulation and morphometrical reactions indicate no major effects. J Steroid Biochem Mol Biol 98:155-163.

OECD (2007) OECD test guideline 440 uterotrophic bioassay in rodents: a short-term screening test for oestrogenic properties.

OECD (2008) Draft OECD guideline for the testing of chemicals. The Hershberger bioassay in rats: A short term test for (anti) androgenic properties.

Pivnenko K, Pedersen GA, Eriksson E, Astrup TF (2015) Bisphenol A and its structural analogues in household waste paper. Waste Manag 44:39-47.

Richter CA, Birnbaum LS, Farabollini F, Newbold RR, Rubin BS, Talsness CE, Vandenbergh JG, Walser-Kuntz $\mathrm{DR}$, vom Saal FS (2007) In vivo effects of bisphenol A in laboratory rodent studies. Reprod Toxicol 24:199224.
Rochester JR, Bolden AL (2015) Bisphenol S and F: A systematic review and comparison of the hormonal activity of bisphenol a substitutes. Environ Health Perspect 123:643-650.

Rubin BS (2011) Bisphenol A: An endocrine disruptor with widespread exposure and multiple effects. J Steroid Biochem Mol Bio 127:27-34.

Sifakis S, Androutsopoulos VP, Tsatsakis AM, Spandidos DA (2017) Human exposure to endocrine disrupting chemicals: Effects on the male and female reproductive systems. Environ Toxicol Pharmacol 51:56-70.

Teng C, Goodwin B, Shockley K, Xia M, Huang R, Norris J, Merrick BA, Jetten AM, Austin CP, Tice RR (2013) Bisphenol A affects androgen receptor function via multiple mechanisms. Chem Biol Interact 203:556-564.

WHO (2013) State of the science of endocrine disrupting chemicals - 2012. World Health Organization. Retrieved 2015-04-06.

Yoon K, Kwack SJ, Kim HS, Lee BM (2014) Estrogenic endocrine-disrupting chemicals: molecular mechanisms of actions on putative human diseases. J Toxicol Environ Health B Crit Rev 17:127-174. 